\title{
An inversion approach for determining distribution of production and temperature sensitivity of soil respiration
}

\author{
Robyn N. C. Latimer and David A. Risk \\ St. Francis Xavier University, P.O. Box 5000, Antigonish, Nova Scotia, B2G 2W5, Canada \\ Correspondence to: David A. Risk (drisk@stfx.ca)
}

Received: 8 May 2015 - Published in Biogeosciences Discuss.: 8 July 2015

Revised: 8 February 2016 - Accepted: 11 February 2016 - Published: 11 April 2016

\begin{abstract}
Physical soil properties create lags between temperature change and corresponding soil responses, which obscure true $\mathrm{Q}_{10}$ (temperature sensitivity) values and other biophysical parameters such as depth of production. This study examines an inversion approach for estimating $\mathrm{Q}_{10}$ and $e$ folding depth of $\mathrm{CO}_{2}$ production $\left(Z_{\mathrm{p}}\right)$ using physically based soil models, constrained by observed high-frequency surface fluxes and/or concentrations. Our inversion strategy uses a one-dimensional (1-D) multi-layered soil model that simulates realistic temperature and gas diffusion. We tested inversion scenarios on synthetic data using a range of constraining parameters, time-averaging techniques, mechanisms to improve computational efficiency, and various methods of incorporating real data into the model. Overall, we have found that with carefully constrained data, inversion was possible. While inversions using exclusively surface-flux measurements could succeed, constraining the inversion using multiple shallow subsurface $\mathrm{CO}_{2}$ measurements proved to be most successful. Inversions constrained by these shallow measurements returned $\mathrm{Q}_{10}$ and $Z_{\mathrm{p}}$ values with average errors of 1.85 and $0.16 \%$ respectively. This work is a first step toward building a reliable framework for removing physical effects from high-frequency soil $\mathrm{CO}_{2}$ data. Ultimately, we hope that this process will lead to better estimates of biophysical soil parameters and their variability on short timescales.
\end{abstract}

\section{Introduction}

Soil respiration, which includes both root and microbial respiration, represents the largest outward flux of $\mathrm{CO}_{2}$ from terrestrial ecosystems, with a magnitude far above that of anthropogenic emissions (Raich et al., 2002). Small changes in the soil $\mathrm{CO}_{2}$ flux could therefore have a significant impact on the carbon balance and global atmospheric $\mathrm{CO}_{2}$ concentrations. In predictions of atmospheric $\mathrm{CO}_{2}$ over the 21st century, uncertainties surrounding the response of land flux to climate change are second only to uncertainties surrounding future anthropogenic emissions (Meir et al., 2006). In order to accurately predict future atmospheric $\mathrm{CO}_{2}$ concentrations, it is crucial to gain a better understanding of how land systems will respond to changing temperature and moisture regimes.

Soil $\mathrm{CO}_{2}$ production originates from plant root respiration and microbial decomposition of organic matter. The temperature sensitivity of soil respiration describes how the flux of $\mathrm{CO}_{2}$ from soils will respond to a change in temperature. Normally soil microbial and plant root processes are treated together because they are not readily distinguished from one another. Temperature sensitivity is often quantified by the parameter $\mathrm{Q}_{10}$ (temperature sensitivity), which describes the factor increase in soil respiration with a temperature increase of $10^{\circ} \mathrm{C}$. This $\mathrm{Q}_{10}$ parameter is used in global climate models to quantify soil feedbacks to climate change. It has been found that $\mathrm{Q}_{10}$ values are influenced by a range of environmental factors including soil temperature (Lloyd and Taylor, 1994; Luo et al., 2001), soil volumetric water content (Davidson et al., 1998; Reichstein et al., 2002), and soil organic matter content (Taylor et al., 1989; Wan and Luo, 2003). As these factors exhibit high spatial heterogeneity across ecosystems as well as within a given ecosystem, it has long been expected that $\mathrm{Q}_{10}$ will also exhibit high spatial variability. Despite this, most existing models continue to use a globally constant $\mathrm{Q}_{10}$ value. This may reduce or enhance predicted release of $\mathrm{CO}_{2}$ from soils, leading to large over- or under-estimates of the contribution of soil respiration to terrestrial $\mathrm{CO}_{2}$ flux in 


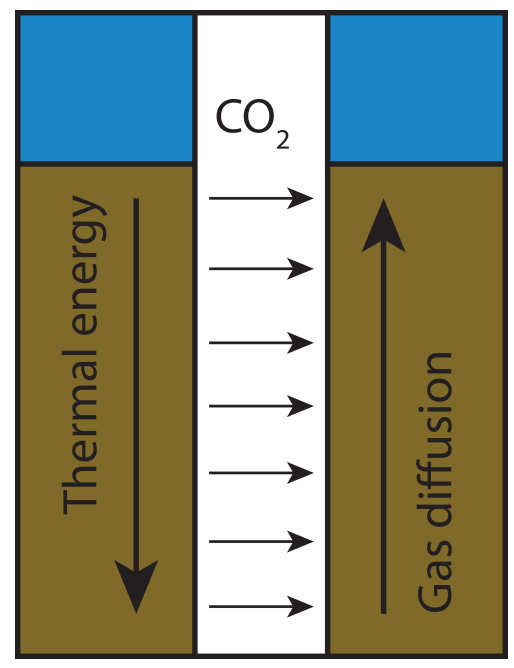

Figure 1. Thermal and gas diffusion lags through a soil profile.

the face of climate change. There has been considerable debate over the usage and magnitude of $\mathrm{Q}_{10}$ (Davidson et al., 2006; Mahecha et al., 2010), with different studies producing widely variant values. While most studies agree that $\mathrm{CO}_{2}$ flux feedback will be positive, there is no consensus on how best to estimate the magnitude of $\mathrm{Q}_{10}$.

Historically, $\mathrm{Q}_{10}$ values have been determined through regression analysis of soil temperature and $\mathrm{CO}_{2}$ surface-flux measurements. A known source of error in this approach originates in the physics of soil heat and gas transport, which might separate a change in surface soil temperature (normally a 5 or $10 \mathrm{~cm}$ temperature is used for deriving $\mathrm{Q}_{10}$ ) from the resultant change in $\mathrm{CO}_{2}$ flux measured at the surface. The lags depend most heavily on soil heat transport (Phillips et al., 2011), because changes in surface temperature are shifted and dampened significantly as a function of depth, with each successive soil layer experiencing a reduced temperature change in amplitude. Gas diffusion also plays an important role, and even if soil microbes and roots produced $\mathrm{CO}_{2}$ instantaneously upon receipt of thermal energy at the characteristic production depths, gases still take time to diffuse upward. Soil properties including heat and gas diffusion, and the $e$-folding depth of $\mathrm{CO}_{2}$ production $\left(Z_{\mathrm{p}}\right)$, all contribute to these lags (Fig. 1). Phillips et al. (2011) demonstrated that such lags can lead to severe misinterpretation of data when attempting to extract true $\mathrm{Q}_{10}$ values through regression of surface flux and a temperature measurement at a single depth.

These thermal and gas diffusion processes, and the resulting lags, can be captured in a simple one-dimensional (1-D) physical heat and gas transport soil model (Nickerson and Risk, 2009; Phillips et al., 2011). Though not done to date for the soil respiration system, it is possible to use such a model in inverse fashion for estimating the value of parameters like $\mathrm{Q}_{10}$ and $Z_{\mathrm{p}}$ by looping the forward model iter- atively through possible parameter combinations, with observed measurements as a constraint. Normally, an objective function is used for helping decide which parameter set best minimizes the difference between modelled and measured data. This method has been identified as a promising tool for determining unknown soil parameters (Zhou et al., 2009), with an increasing availability of high-frequency data sets allowing for rigorous constraints on known model parameters.

This study seeks to develop a reliable inversion framework for determining the $\mathrm{Q}_{10}$ and $Z_{\mathrm{p}}$ of different sites given continuous soil measurements. It also seeks to provide guidance for researchers who would like to build field observational sites suited for inversion analysis. Working exclusively with synthetic soil data that mimics the form of collected field data and of which all parameters are known, we first undertake sensitivity tests to determine optimal sensor placing in the field, and decide whether soil $\mathrm{CO}_{2}$ surface flux, and/or profile measurements, are more suited for anchoring inversion approaches with the necessary field data for parameter constraint. Using the best sensor combination, we are able to evaluate the accuracy of the inversion approach in returning the original $\mathrm{Q}_{10}$, and $Z_{\mathrm{p}}$, across many realistic soil type scenarios.

\section{Methods}

This study uses a 1-D $\mathrm{CO}_{2}$ and heat transport model described by Phillips et al. (2011), originally developed by Nickerson and Risk (2009). This model, with existing versions in Perl and R (R Core Team, 2015), was recoded in C to increase computational efficiency for the parameter solving routine.

\subsection{Model description}

This model (Fig. 2) simulates the movement and production of $\mathrm{CO}_{2}$ through the soil profile and into the free atmosphere. The model consists of one atmospheric layer and a soil profile $1 \mathrm{~m}$ in length, divided into 100 layers of uniform thickness. Each layer can exchange $\mathrm{CO}_{2}$ with its two nearest neighbouring layers using the 1-D discrete form of Fick's first law:

$F_{i j}=-D_{i j} \frac{\Delta C_{i j}}{\Delta z_{i j}}$,

where $D_{i j}$ is the effective diffusion coefficient between two soil layers, $\Delta C_{i j}$ is the $\mathrm{CO}_{2}$ concentration difference $\left(\mu \mathrm{mol} \mathrm{m}{ }^{-3}\right)$ and $\Delta z_{i j}$ is the difference in depth $(\mathrm{m})$ between the two layers.

For every modelled time step, each soil layer has a defined temperature, biological $\mathrm{CO}_{2}$ production, $\mathrm{CO}_{2}$ flux, thermal diffusivity, and gas diffusivity. Temperature varies sinusoidally on daily and annual timescales. Changes in surface temperature are shifted and dampened through the soil 


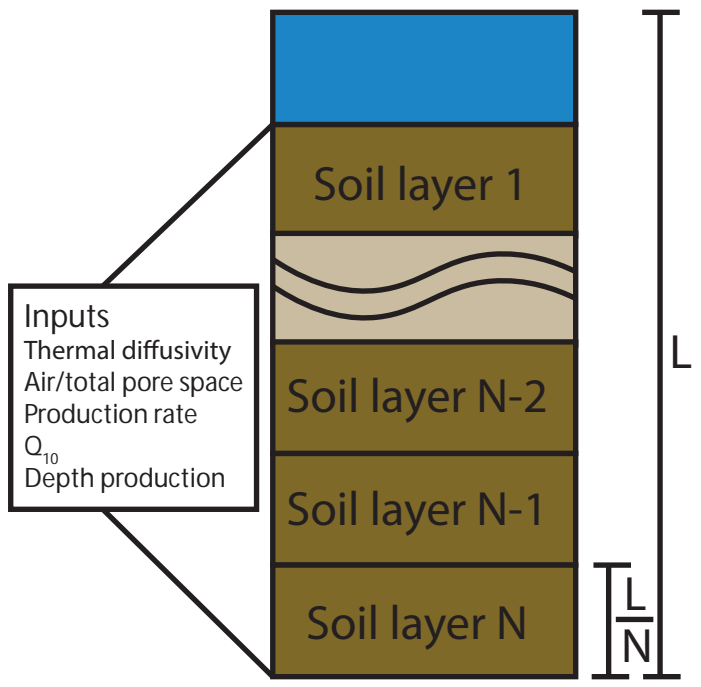

Figure 2. Conceptual representation of the 1-D layered soil model. Overall profile length is denoted with $L$, and $N$ represents the number of individual layers in the model soil profile.

profile using

$$
\begin{aligned}
T[i] & =T_{\mathrm{avg}}+\Delta T_{\mathrm{D}} e^{\frac{-z_{i}}{T_{\mathrm{D}}}} \sin \left(\omega_{\mathrm{D}} t-\frac{z_{i}}{d_{T_{\mathrm{D}}}}\right) \\
& +\Delta T_{\mathrm{Y}} e^{\frac{-z_{i}}{d_{\mathrm{Y}}}} \sin \left(\omega_{\mathrm{Y}} t-\frac{z_{i}}{d_{T_{\mathrm{Y}}}}\right), \\
d_{T_{\mathrm{D}}} & =\sqrt{\frac{2 D_{\mathrm{T}}[i]}{\omega_{\mathrm{D}}}}, d_{T_{\mathrm{Y}}}=\sqrt{\frac{2 D_{\mathrm{T}}[i]}{\omega_{\mathrm{Y}}}},
\end{aligned}
$$

which simulates the lags related to the rates of thermal diffusion. In this equation, $T_{\text {avg }}$ is the average temperature in the air and soil profile for the duration of the simulation, $\Delta T_{\mathrm{D}}$ is the amplitude of the daily temperature fluctuation, $\Delta T_{\mathrm{Y}}$ is the amplitude of the yearly temperature fluctuation, $\omega_{\mathrm{D}}$ is the radial frequency for daily oscillations $\left(\omega_{\mathrm{D}}=2 \pi / 86400 \mathrm{~s}\right)$, $\omega_{\mathrm{Y}}$ is the radial frequency for annual oscillations, $z_{i}$ is the layer depth (m), and $D_{\mathrm{T}}$ is the thermal diffusivity of the soil $\left(\mathrm{m}^{2} \mathrm{~s}^{-1}\right)$.

Biological $\mathrm{CO}_{2}$ production in each layer is calculated using an exponentially decreasing function (Nickerson and Risk, 2009):

$P[i]=\frac{\Gamma_{0}}{\sum_{i=1}^{N} e^{\frac{-z_{i}}{Z_{\mathrm{p}}}}} e^{\frac{-z_{i}}{Z_{\mathrm{p}}}} Q_{10}^{\frac{T[i]-T_{\mathrm{avg}}}{10}}$,

where $\Gamma_{0}$ is the total basal soil production $\left(\mu \mathrm{mol} \mathrm{m}^{-3} \mathrm{~s}^{-1}\right)$, $N$ is the number of soil layers, $\mathrm{Q}_{10}$ is the temperature sensitivity of soil respiration, $z_{i}$ is the depth of the layer $(\mathrm{m})$, and $Z_{\mathrm{p}}$ is the $e$-folding depth of $\mathrm{CO}_{2}$ production $(\mathrm{m})$, defined as the depth below which the total fraction of $\mathrm{CO}_{2}$ production remaining is $1 / e$ (also called the characteristic production depth in some studies), from which the production at any depth $P(i)$ can be calculated based on Eq. (4).
Initially, the diffusivity of $\mathrm{CO}_{2}$ in the soil profile is calculated using the Millington model (Millingon, 1959), an empirically derived approximation for calculating diffusivity in the field:

$D_{c}=\frac{\theta_{\mathrm{w}}^{\frac{10}{3}} \frac{D_{\mathrm{fw}}}{H}+\theta_{\mathrm{g}}^{\frac{10}{3}} D_{\mathrm{fg}}}{\theta_{\mathrm{T}}^{2}}$,

where $D_{\mathrm{fw}}$ and $D_{\mathrm{fg}}$ are the diffusivity of $\mathrm{CO}_{2}$ in free water and free air $\left(\mathrm{m}^{2} \mathrm{~s}^{-1}\right), H$ is the dimensionless form of Henry's solubility constant for $\mathrm{CO}_{2}$ in water, and $\theta_{\mathrm{w}}, \theta_{\mathrm{g}}$, and $\theta_{\mathrm{T}}$ are the water filled, air filled, and total soil porosities, respectively.

At each time step, the diffusivity of each soil layer is calculated using a temperature correction on this Millington diffusivity:

$D[i]=D_{c}\left(\frac{T[i]}{T_{\text {avg }}}\right)^{1.75}$.

As previously mentioned, the flux from each layer is determined by Fick's first law, written explicitly as

$F[i]=D[i] \frac{(C[i]-C[i-1])}{\mathrm{d} z} \mathrm{~d} t$,

where $C[i]$ is the $\mathrm{CO}_{2}$ concentration of layer $i\left(\mu \mathrm{mol} \mathrm{m}{ }^{-3}\right)$, $C[i-1]$ is the concentration of the layer above, and $\mathrm{dt}$ is the time step $(s)$.

Finally, at each time step $\mathrm{CO}_{2}$ concentration in each layer $i$ is calculated using

$C[i]=\frac{C_{t-1}[1] \theta_{\mathrm{g}} \mathrm{d} z-F[i]+F[i+1]+P[i]}{\theta_{\mathrm{g}} \mathrm{d} z}$,

where $C_{t-1}[\mathrm{i}]$ is the layer concentration at the previous time step, $F[i]$ is the flux of $\mathrm{CO}_{2}$ leaving layer $i, F[i+1]$ is the flux of $\mathrm{CO}_{2}$ entering the layer from the layer below, and $P[i]$ is the $\mathrm{CO}_{2}$ production within layer $i$.

\subsection{Model execution and validation}

Before beginning the simulation, the system is initialized using input parameters seen in Table 1. Atmospheric $\mathrm{CO}_{2}$ concentration remains constant for the duration of the simulation; it is assumed that any flux from the soil will quickly dissipate into the atmosphere. Flux from the bottom soil boundary is set to zero, as production at this depth is negligible according to the exponentially decreasing production function. These system parameters were changed depending on the soil type being simulated.

After initialization, the system undergoes spin-up, during which layer temperatures are held constant at their initial values, and the model is run until the $\mathrm{CO}_{2}$ concentration in each layer is constant. The duration of the spin-up period is dependent on soil diffusivity (and therefore $\theta_{\mathrm{w}}$ ), and is determined 
Table 1. Default parameter values for simulations.

\begin{tabular}{ll}
\hline Parameter & Value/range \\
\hline Soil porosity $\left(\theta_{\mathrm{T}}\right)$ & $0.40(v / v)$ \\
Thermal diffusivity $\left(D_{\mathrm{T}}\right)$ & $5 \times 10^{-7}\left(\mathrm{~m}^{2} \mathrm{~s}^{-1}\right)$ \\
Average air and soil temperature $\left(T_{\mathrm{avg}}\right)$ & $15^{\circ} \mathrm{C}$ \\
Daily air temperature amplitude $\left(\Delta T_{\mathrm{D}}\right)$ & $5^{\circ} \mathrm{C}$ \\
Yearly air temperature amplitude $\left(\Delta T_{\mathrm{Y}}\right)$ & $12^{\circ} \mathrm{C}$ \\
Atmospheric $\mathrm{CO}_{2}$ & $380 \mathrm{ppm}$ \\
Total basal $\mathrm{CO}_{2}$ production $\left(\Gamma_{0}\right)$ & $1-10 \mu \mathrm{mol} \mathrm{m}^{-2} \mathrm{~s}^{-1}$ \\
Production exponential folding depth $\left(Z_{\mathrm{p}}\right)$ & $0.05-0.20 \mathrm{~m}$ \\
$\mathrm{Q}_{10}$ & $1.5-4.5$ \\
Volumetric water content $\left(\theta_{\mathrm{w}}\right)$ & $0.10-0.25(v / v)$ \\
\hline
\end{tabular}

by plotting concentration vs. time through the soil profile. This period ranges from 5 to 23 model days within the range of $\theta_{\mathrm{w}}(0.1$ to 0.25$)$. The $\mathrm{CO}_{2}$ concentration in each layer after spin-up is the initial layer concentration at the beginning of the actual simulation.

For each modelled time step $(\mathrm{d} t=1.0 \mathrm{~s})$, temperature, $\mathrm{CO}_{2}$ diffusivity, $\mathrm{CO}_{2}$ production, and $\mathrm{CO}_{2}$ flux are calculated in each soil layer. Every soil layer is then revisited, and the new $\mathrm{CO}_{2}$ layer concentrations are calculated. The progress of the simulation is monitored by outputting the $\mathrm{CO}_{2}$ concentration and temperature of specified layers.

\subsubsection{Validation}

To ensure the model was performing correctly, steadystate concentrations through depth (following spin-up) were compared to the steady-state solution proposed by Cerling (1984). Daily and yearly temperature fluctuations were removed from the model, and the model was run until $\mathrm{CO}_{2}$ concentrations in each layer were constant. Deviations of modelled from analytic concentrations were found to be far less than $1 \%$.

\subsection{Incorporating constraining data}

In this study, we incorporated external data in the same way we would with field studies. We started with real measurements of temperature through depth, and soil volumetric water content, from a local field site. One of the largest challenges in preparing data for inversion is to accurately model soil temperature through depth and time, as temperature is the known determinant of soil lags (Phillips et al., 2011). For each set of temperature measurements through depth, a linear regression (in $R$ ) was performed, resulting in a fifth-order polynomial for temperature through depth every $1800 \mathrm{~s}$. A linear interpolation through time was performed to obtain temperature values in each layer for every modelled time step. The resultant temperature values replaced our originally sinusoidally varying temperature function in the model. The value of thermal diffusivity was therefore implicitly built into these measurements and is no longer required as a direct model input.

These physical variables were used in a forward instance of the soil model to create $\mathrm{CO}_{2}$ surface-flux and $\mathrm{CO}_{2}$ concentration time series. Data sets were created using many $Z_{\mathrm{p}}$ and $\mathrm{Q}_{10}$ values of interest, so that we had many idealized data sets on hand in which concentration, fluxes, and associated temperatures, $Z_{\mathrm{p}}$, and $\mathrm{Q}_{10}$ values were known. Soil volumetric water content was not formally incorporated as a driver of respiration in these synthetic data sets, so all simulations were performed over periods of constant soil volumetric water content. During inversion we pretended not to know $Z_{\mathrm{p}}$ and $Q_{10}$ values of these synthetic data sets, and hoped the inversion process would return the known values. Since the same forward soil model that generated the synthetic data sets was also embedded within the inversion scheme, errors in $Z_{\mathrm{p}}$, or $\mathrm{Q}_{10}$, would be due entirely to the inversion process itself.

\subsection{Inversion process}

The soil profile $\mathrm{CO}_{2}$ concentrations and soil $\mathrm{CO}_{2}$ surface flux are outputs of the simulation. Their values are dependent on all of the system input parameters. A method called inverse parameter estimation is employed to determine the values of $\mathrm{Q}_{10}$ and $Z_{\mathrm{p}}$ that would have given rise to the observed concentrations and fluxes. Through this process, model outputs are compared to measured field data or synthetic data over a range of model input parameters. The field measurements used in this process will be referred to as the model constraints; these constraints consist of $\mathrm{CO}_{2}$ concentration measurements at various depths in the soil profile, as well as $\mathrm{CO}_{2}$ surface-flux measurements.

\subsubsection{Inversion steps}

The model is run for two unknowns, including $\mathrm{Q}_{10}$ values ranging from 1 to 5.5 in steps of 0.1 , and $Z_{\mathrm{p}}$ from 0.02 to $0.3 \mathrm{~m}$ in steps of $0.01 \mathrm{~m}$. This results in a total of 1260 parameter combinations. Inversion seeks to identify the parameter set that minimizes the objective function

$\sqrt{\left(S_{1}-M_{1}\right)^{2}+\left(S_{2}-M_{2}\right)^{2}+\left(S_{3}-M_{3}\right)^{2}+\ldots}$,

where $S_{i}$ and $M_{i}$ correspond to modelled and measured $\mathrm{CO}_{2}$ concentrations at various profile depths. For each parameter set, this objective function is calculated every 1800 time steps and averaged at the end of the simulation. The pair that minimizes Eq. (9) is output as the inversion result.

\subsection{Validation of the inverse method}

Before applying the inversion method to real field data, tests must be done to ensure method accuracy, and this manuscript focuses on such tests. We created synthetic time series using the original soil model, that mimic the form of real data 
sets. The values of $\mathrm{Q}_{10}$ and $Z_{\mathrm{p}}$ were known for each synthetic time series, as these parameters are required to run the model. This synthetic data included temperature measurements at six depths in the profile, volumetric water content, $\mathrm{CO}_{2}$ surface-flux, and $\mathrm{CO}_{2}$ concentration measurements at various depths in the soil profile.

The inverse method was applied to these synthetic data sets, and the output value of $\mathrm{Q}_{10}$ and $Z_{\mathrm{p}}$ could then be compared to the actual values of these parameters used to create the time series.

\subsubsection{Constraint, sensitivity, and random error testing}

To determine which model constraints resulted in the highest accuracy of the inversion method, the error (Eq. 9) was calculated using a large range of constraining parameters and combinations thereof. A total of 35 different constraint combinations were tested, representing various combinations of surface $\mathrm{CO}_{2}$ flux, and subsurface $\mathrm{CO}_{2}$ concentration measurements up to $0.6 \mathrm{~m}$ depth. These combinations are illustrated in Table 2. Testing which constraints consistently returned the most accurate values of $\mathrm{Q}_{10}$ and $Z_{\mathrm{p}}$ aids in determining optimal sensor placing in the field.

To ensure model validity across all possible parameter values that may be encountered in the field, extensive sensitivity testing was done using these synthetic time series. These time series were created across a range of combinations of $\mathrm{Q}_{10}, Z_{\mathrm{p}}$, volumetric water content (diffusivity), and total soil production. Table 3 illustrates the ranges tested for each parameter.

Field-deployable $\mathrm{CO}_{2}$ sensors typically have $1-5 \%$ error. To see how the model and inversion would perform under these conditions, errors of 1,5 , and $10 \%$ were added into all components of the synthetic data. The effect of these errors on the inverse method were observed.

\section{Results and discussion}

Inversions on synthetic time series were successful across all tested soil parameters, though some $\mathrm{CO}_{2}$ concentration measurement depth combinations (surface flux, single, or multiple profile measurements) helped to minimize the overall error, as well as the error in $\mathrm{Q}_{10}$ and $Z_{\mathrm{p}}$ individually. Errors discussed in this section represent an average from 64 inversions across values of $\mathrm{Q}_{10}, Z_{\mathrm{p}}$, and soil diffusivity as presented in Table 3. In this section, we use either fractional error $\left(\frac{\text { actual-result } \mid}{\text { actual }}\right)$, or absolute deviation from the actual value (|actual-result $\mid)$.

\subsection{Best measurement configurations to obtain $Q_{10}$ and $Z_{p}$ via inversion}

In Fig. 3 we show the average fractional error in the returned $\mathrm{Q}_{10}$ value for every combination of subsurface $\mathrm{CO}_{2}$ sensor measurements. Observations of $\mathrm{CO}_{2}$ concentration shallow in the soil were found to be necessary for highly accurate $\mathrm{Q}_{10}$ estimates. The lowest inversion error for $\mathrm{Q}_{10}$ was $1.85 \%$, in a scenario where subsurface measurements were made at 5,10 , and $15 \mathrm{~cm}$. Single concentration measurements at or above $10 \mathrm{~cm}$ also proved successful, with errors $<2.3 \%$. The least accurate inversions for $\mathrm{Q}_{10}$ occurred when the constraint consisted of (single or multiple) $\mathrm{CO}_{2}$ concentration measurements deep in the soil profile. We propose that the poor performance of inversion when using deep profile constraints could be related to the low magnitude of thermal and concentration variability at these depths. Deep soil layers are subject to much smaller thermal fluctuations than layers close to the surface. In this less variable environment, $\mathrm{CO}_{2}$ concentrations are less variable and provide less of a signal upon which to anchor inversion. In contrast, $\mathrm{CO}_{2}$ concentrations shallow in the soil exhibited larger variations in temperature and concentration, which presumably allowed $\mathrm{Q}_{10}$ to be extracted more easily. If the primary interest is to obtain $\mathrm{Q}_{10}$ from inversion, multiple $\mathrm{CO}_{2}$ concentration measurements in the soil were found to be important. It should be noted that, while differences in error rate were noted, errors for all scenarios could be considered tolerably low relative to the normal variance expected from regression-based $\mathrm{Q}_{10}$, considering the gas transport lags inherent in those data (Phillips et al., 2011).

The average fractional error in $Z_{\mathrm{p}}$ for different model sensor combination constraints is also shown in Fig. 3. Out of the 35 combinations tested, only 5 resulted in an average $Z_{p}$ error greater than $2 \%$. Single concentration measurements shallow or deep in the soil profile caused this larger error, but on average, single concentration measurements at any depth in the soil were less accurate. Inversions constrained by at least one measurement shallow $(<15 \mathrm{~cm})$ and one deep $(\geq 30 \mathrm{~cm})$ in the profile returned $Z_{\mathrm{p}}$ with $100 \%$ accuracy across all sensitivity tests. We did expect that single measurements deep in the profile would perform poorly relative to others, because with the exponentially decreasing production defined in the model, $\mathrm{CO}_{2}$ production approaches zero at significant depths regardless of the value of $Z_{\mathrm{p}}$ and thus cannot perform well as an inversion anchor. The large $Z_{\mathrm{p}}$ error of almost $25 \%$ associated with soil surface $\mathrm{CO}_{2}$-flux measurements, was also not surprising. In this situation the inversion scheme must reconstruct $Z_{\mathrm{p}}$ mainly via the temporal delay, and damping, between sinusoids of temperature through depth, and soil surface $\mathrm{CO}_{2}$ flux. Without a concentration measurement in the soil, the gas transport regime is black boxed from the perspective of the inversion scheme, resulting in the large error. Overall, surface $\mathrm{CO}_{2}$-flux measurements alone are less suited for elucidating information on $e$-folding depth of production, whereas a combination of shallow and deep measurements is best for reconstructing the distribution of $\mathrm{CO}_{2}$ production in the soil profile.

In examining inversion accuracy for both parameters $Q_{10}$ and $Z_{\mathrm{p}}$ simultaneously (Fig. 3), we found that multiple concentration measurements shallow in the soil $(\leq 15 \mathrm{~cm})$, or 
Table 2. Measurement combinations used for the simulations. The combination number is listed at the beginning of each row. The columns represent the type of measurement (e.g. $\mathrm{CO}_{2}$ surface flux), or the depth of concentration measurement in centimetres. The " $\times$ " values denote whether the type or depth of measurement was included in the combination.

\begin{tabular}{|c|c|c|c|c|c|c|c|c|c|c|c|c|c|}
\hline Combination & Flux & 5 & 10 & 15 & 20 & 25 & 30 & 35 & 40 & 45 & 50 & 55 & 60 \\
\hline 1 & $x$ & & & & & & & & & & & & \\
\hline 2 & & $x$ & & & & & & & & & & & \\
\hline 3 & & & $x$ & & & & & & & & & & \\
\hline 4 & & & & $\times$ & & & & & & & & & \\
\hline 5 & & & & & $x$ & & & & & & & & \\
\hline 6 & & & & & & $\times$ & & & & & & & \\
\hline 7 & & & & & & & $x$ & & & & & & \\
\hline 8 & & & & & & & & $\times$ & & & & & \\
\hline 9 & & & & & & & & & $\times$ & & & & \\
\hline 10 & & & & & & & & & & $x$ & & & \\
\hline 11 & & & & & & & & & & & $\times$ & & \\
\hline 12 & & & & & & & & & & & & $x$ & \\
\hline 13 & & & & & & & & & & & & & $x$ \\
\hline 14 & & $x$ & & $\times$ & & & & & & & & & \\
\hline 15 & & $x$ & & & & & $x$ & & & & & & \\
\hline 16 & & $x$ & & & & & & & & & $\times$ & & \\
\hline 17 & & $x$ & & & & & & & & & & & $x$ \\
\hline 18 & & & & $\times$ & & & $x$ & & & & & & \\
\hline 19 & & & & $\times$ & & & & & & & $x$ & & \\
\hline 20 & & & & $\times$ & & & & & & & & & $x$ \\
\hline 21 & & & & & & & $x$ & & & & $\times$ & & \\
\hline 22 & & & & & & & $x$ & & & & & & $x$ \\
\hline 23 & & & & & & & & & & & $\times$ & & $x$ \\
\hline 24 & & $x$ & & $\times$ & & & $x$ & & & & & & \\
\hline 25 & & $x$ & & $\times$ & & & & & & & $\times$ & & \\
\hline 26 & & $x$ & & $\times$ & & & & & & & & & $x$ \\
\hline 27 & & & & $\times$ & & & $x$ & & & & $\times$ & & \\
\hline 28 & & & & $\times$ & & & $x$ & & & & & & $x$ \\
\hline 29 & & & & & & & $x$ & & & & $\times$ & & $x$ \\
\hline 30 & & $x$ & & $\times$ & & & $x$ & & & & $\times$ & & \\
\hline 31 & & $x$ & & $\times$ & & & $x$ & & & & & & $x$ \\
\hline 32 & & & & $\times$ & & & $x$ & & & & $x$ & & $x$ \\
\hline 33 & & $x$ & $x$ & $\times$ & & & & & & & & & \\
\hline 34 & & & & & & $\times$ & $x$ & $\times$ & & & & & \\
\hline 35 & & & & & & & & & & & $\times$ & $x$ & $x$ \\
\hline
\end{tabular}

Table 3. Default parameter values for sensitivity testing.

\begin{tabular}{lrrrr}
\hline Parameter & Abbr. & Minimum & Maximum & Increment \\
\hline Total basal $\mathrm{CO}_{2}$ production $\left(\mu \mathrm{mol} \mathrm{m} \mathrm{m}^{-2} \mathrm{~s}^{-1}\right)$ & $\Gamma_{0}$ & 1 & 10 & 10 \\
Production exponential folding depth $(\mathrm{m})$ & $Z_{\mathrm{p}}$ & 0.05 & 0.2 & 0.05 \\
$\mathrm{Q}_{10}$ & & 1.5 & 4.5 & 1 \\
Volumetric water content $(v / v)$ & $\theta_{\mathrm{w}}$ & 0.1 & 0.25 & 0.05 \\
\hline
\end{tabular}

combinations shallow in the soil with one deep concentration measurement $(\geq 30 \mathrm{~cm}$ ) were the best constraints. Deep soil measurements and surface-flux constraints should therefore be avoided if the aim is to minimize overall error. This overall result is a combination of what was found for $\mathrm{Q}_{10}$ and $Z_{\mathrm{p}}$ individually, where shallow measurements were best for
$\mathrm{Q}_{10}$ and a combination of shallow and deep measurements resulted in most accurate $Z_{\mathrm{p}}$.

Depending on error tolerance for the final parameter estimates, it is conceivable that the accuracy of all inversions performed here might be sufficient for the community of soil scientists. Out of the 35 combinations tested, 19 resulted in 

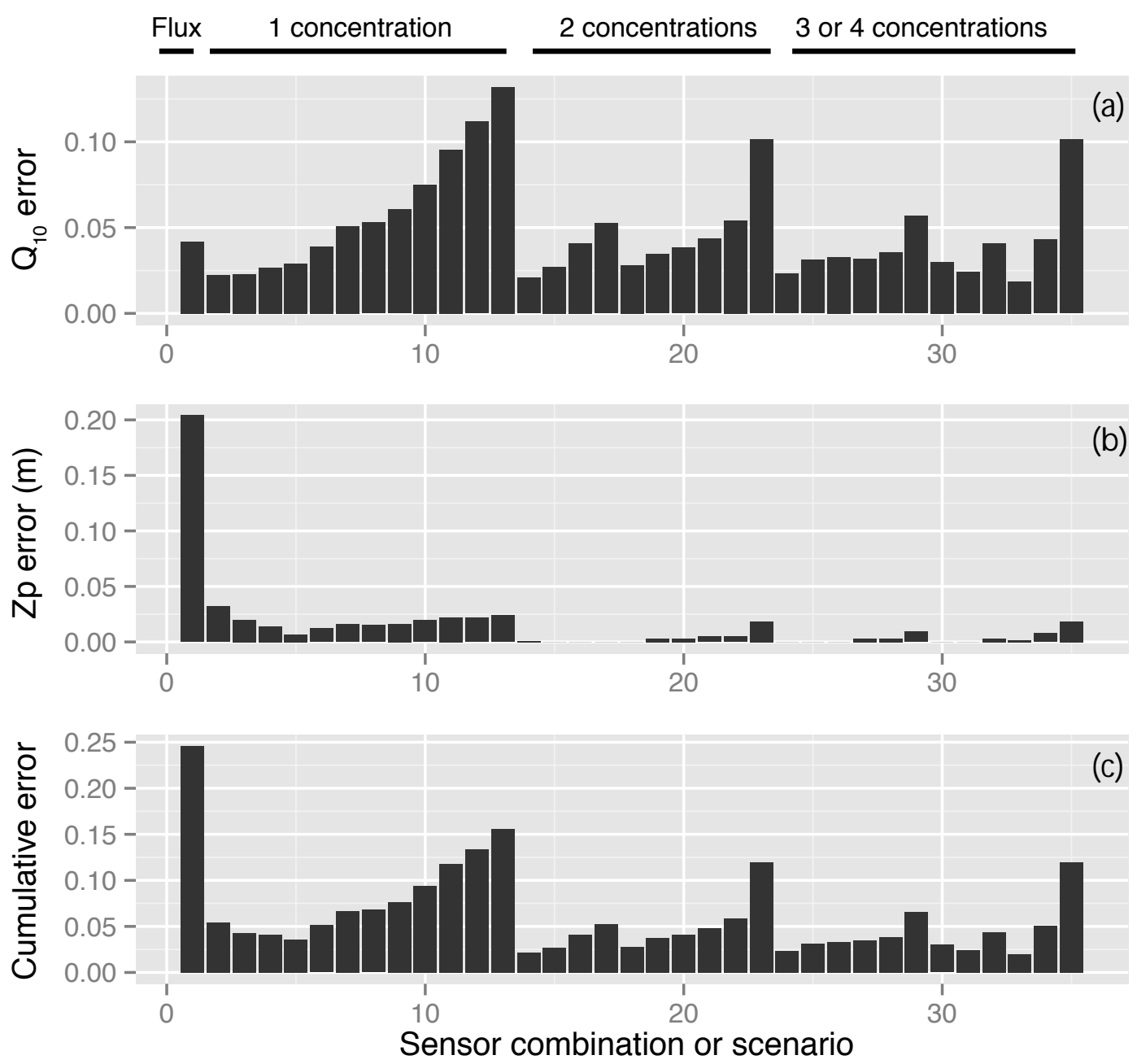

Figure 3. Fractional error in $\mathrm{Q}_{10}$ and $Z_{\mathrm{p}}$ individually for different sensor combination scenarios, plus cumulative fractional error in $\mathrm{Q}_{10}$ and $Z_{\mathrm{p}}$ for the same scenarios.

an overall average error less than $5 \%$. The top constraint (measurements at 5,10, and $15 \mathrm{~cm}$ ) had an average error of $2.01 \%$, and the top six combinations all had error less than $3 \%$. These errors are small compared to the degree of random error in $\mathrm{CO}_{2}$-flux studies (Lavoie et al., 2015). These results are summarized in Table 4, where the top and bottom five combinations are listed individually and overall.

This assessment was performed using synthetic data, and even the most ideal field settings will depart from these modelled profiles. For example, we represented $\mathrm{CO}_{2}$ production through depth using an exponential production function, but a field site may show a linear decrease in production at increasing depths. Clearly users of the inversion process will want to characterize as many site-specific parameters as possible so as to provide proper guideposts and constraints for the inversion; otherwise, additional error will be introduced. The sensitivity of the inversion to error is an important question, and will be addressed in a later section.
Table 4. Best and worst sensor combinations for determining $\mathrm{Q}_{10}$, $Z_{\mathrm{p}}$, and overall through inversion.

\begin{tabular}{llll}
\hline & \multicolumn{3}{c}{ Combination } \\
\cline { 2 - 4 } Rank & $\mathrm{Q}_{10}$ & $Z_{\mathrm{p}}$ & Overall \\
\hline 1 & $5+10+15 \mathrm{~cm}$ & $\mathrm{n} / \mathrm{a}$ & $5+10+15 \mathrm{~cm}$ \\
2 & $5+15 \mathrm{~cm}$ & $\mathrm{n} / \mathrm{a}$ & $5+15 \mathrm{~cm}$ \\
3 & $5 \mathrm{~cm}$ & $\mathrm{n} / \mathrm{a}$ & $5+15+30 \mathrm{~cm}$ \\
4 & $10 \mathrm{~cm}$ & $\mathrm{n} / \mathrm{a}$ & $5+15+30+60 \mathrm{~cm}$ \\
5 & $5+15+30 \mathrm{~cm}$ & $\mathrm{n} / \mathrm{a}$ & $5+30 \mathrm{~cm}$ \\
31 & $45 \mathrm{~cm}$ & $55 \mathrm{~cm}$ & $50 \mathrm{~cm}$ \\
32 & $50 \mathrm{~cm}$ & $50 \mathrm{~cm}$ & $50+60 / 50+55+60 \mathrm{~cm}$ \\
33 & $50+60 / 50+55+60 \mathrm{~cm}$ & $60 \mathrm{~cm}$ & $55 \mathrm{~cm}$ \\
34 & $55 \mathrm{~cm}$ & $5 \mathrm{~cm}$ & $60 \mathrm{~cm}$ \\
35 & $60 \mathrm{~cm}$ & Surface Flux & Surface flux \\
\hline
\end{tabular}




\subsection{Effect of soil-specific parameters on inversion success}

Having determined the best $\mathrm{CO}_{2}$ sensor concentration measurement depth to constrain inversions, we can examine how site-specific parameters, such as soil diffusivity, $Z_{\mathrm{p}}$, and $\mathrm{Q}_{10}$, affect inversion results. For this assessment, we will use the best-performing measurement configurations established. Even when not a top choice, we will always include $\mathrm{CO}_{2}$ surface-flux measurements in this section, because of the likelihood that scientists will want to use inversion to analyse these data, which are increasing in number rapidly.

Figure $4 \mathrm{a}$ and $\mathrm{d}$ illustrate how deviation in $\mathrm{Q}_{10}$ and $Z_{\mathrm{p}}$ were affected by the diffusivity of soils. When subsurface sensor combinations were used as a constraint, there was an overall downward trend in $\mathrm{Q}_{10}$ and $Z_{\mathrm{p}}$ error with increasing diffusivity. As diffusivity increases (drier soils), $\mathrm{CO}_{2}$ travels through the soil layers to the surface more quickly, which results in decreased lag times, more rapid concentration changes, and more distinct soil responses. Under these conditions of rapid diffusion, inversions were most successful. Sites that are frequently waterlogged with limited airfilled pore space tended to be less ideal for inversion, but the optimal instrument configuration still helps ensure reasonably small error throughout the entire range of diffusivities, so there is no strict limitation on the use of the inversion approach in low diffusivity soils.

Figure $4 \mathrm{~b}$ and e demonstrate the impact of the $Z_{\mathrm{p}}$ parameter value on inversion success in terms of deviation in returned $\mathrm{Q}_{10}$ and $Z_{\mathrm{p}}$ values. For small $Z_{\mathrm{p}}$ values, shallow $\mathrm{CO}_{2}$ concentration measurements $(\leq 15 \mathrm{~cm})$ were the best constraints, presumably because the soil is most active in these top layers. As $Z_{\mathrm{p}}$ increases, the production of $\mathrm{CO}_{2}$ is no longer limited to the shallow soil, the exponential production function decreases more slowly. With increasing $Z_{\mathrm{p}}$, $\mathrm{CO}_{2}$ production in deeper soil layers is higher, and more useful as an inversion constraint. Some matching of deployment depth was also found, where for example shallow concentration measurements were more accurate for returning the correct value of shallow $\mathrm{CO}_{2}$ production.

Sensitivity tests indicate that increasing the temperature sensitivity of respiration had opposite effects on $\mathrm{Q}_{10}$ and $Z_{\mathrm{p}}$ error. Deviation in returned $\mathrm{Q}_{10}$ values increased rather uniformly across the best subsurface measurements, while for most subsurface combinations the $Z_{\mathrm{p}}$ error decreased. With increasing $\mathrm{Q}_{10}$, respiration becomes more sensitive to temperature changes, leading to larger variations in production in the event of a temperature fluctuation. Figure $4 \mathrm{c}$ and $\mathrm{f}$ illustrate the impact of this parameter on $\mathrm{Q}_{10}$ and $Z_{\mathrm{p}}$ error.

With large amounts of existing surface-flux data, it is also worth examining the effectiveness of the soil $\mathrm{CO}_{2}$ surface flux as a constraint, even when it is not the preferred constraint. It is immediately evident from Fig. 4 that inversions constrained by the surface flux resulted in $\mathrm{Q}_{10}$ and $Z_{\mathrm{p}}$ deviations that responded much differently to changes in soil diffu- sivity, $Z_{\mathrm{p}}$, and $\mathrm{Q}_{10}$. These deviations were often significantly larger than when subsurface constraints were used. Deviations in $\mathrm{Q}_{10}$ and $Z_{\mathrm{p}}$ generally increased as all three parameters increased. This suggests that for low diffusivity, $Z_{\mathrm{p}}$, and $\mathrm{Q}_{10}$, surface flux was a reasonable model constraint, producing errors comparable to the subsurface measurements. This constraint was much less effective for determining depth of $\mathrm{CO}_{2}$ production. However, $Z_{\mathrm{p}}$ was always returned within at least $3.5 \mathrm{~cm}$ of its actual value, which for some uses may be an acceptable level of uncertainty. Inversions constrained by surface flux were quite effective in returning $\mathrm{Q}_{10}$. Returning to Fig. 3, the overall average $\mathrm{Q}_{10}$ error associated with surface flux was less than $5 \%$, which is significantly better than results using deep subsurface measurements. Figure $4 \mathrm{f}$ suggests that inversions using large $\mathrm{Q}_{10}$ values were responsible for the majority of this error. For $\mathrm{Q}_{10}$ of 1.5, these inversions returned $\mathrm{Q}_{10}$ with $100 \%$ accuracy. For the largest $\mathrm{Q}_{10}$, deviation from the true value climbed as high as $0.6-0.7$, which is non-negligible. A shorter model time step could potentially reduce this error, as it may be able to better capture the larger and faster responses associated with high $\mathrm{Q}_{10}$ and diffusivity. As we cannot estimate the $\mathrm{Q}_{10}$ of a site prior to inversion, however, this insight may not be overly useful in site selection. Overall, inversions constrained by the $\mathrm{CO}_{2}$ surface flux are possible but should be performed with caution, and with reasonable expectations as to the resultant error level.

It is also of interest to examine how the amount of $\mathrm{CO}_{2}$ production in the soil profile affects inversion. The bulk of our sensitivity tests were performed using a basal $\mathrm{CO}_{2}$ production of $10 \mu \mathrm{mol} \mathrm{m}^{-3} \mathrm{~s}^{-1}$, which is a fairly high. In order to test the other extreme, several inversions were performed using a production level of $1 \mu \mathrm{mol} \mathrm{m}{ }^{-3} \mathrm{~s}^{-1}$. These inversions performed with exactly the same accuracy as those with a production level of 10 . From this, we can conclude that the magnitude of production has no effect on inversion success.

\subsubsection{Random error and inversion}

The measurements performed by sensors in the field will always be uncertain to some degree. It is therefore important to examine how these uncertainties in recorded temperature, $\mathrm{CO}_{2}$ and soil volumetric water content measurements will impact the accuracy of the inversion method. Inversions performed on synthetic data to which random errors of 1,5 , and $10 \%$ had been added were indeed less accurate than those performed on idealized data. However, the resulting errors in returned $\mathrm{Q}_{10}$ and $Z_{\mathrm{p}}$ were not proportional to the amount of error added to the input data, but actually much lower. That is, errors of $5 \%$ in the input data did not result in an additional $5 \%$ error in output values. An example of this is illustrated in Fig. 5. This plot demonstrates that with random measurement errors in the ranges of $1-5 \%, \mathrm{Q}_{10}$ values were still determined with reasonable accuracy. Prior to error addition, deviation in $\mathrm{Q}_{10}$ was around 0.12 . This deviation increased to 0.14 for $1 \%$ error and 0.17 for $5 \%$ error. 

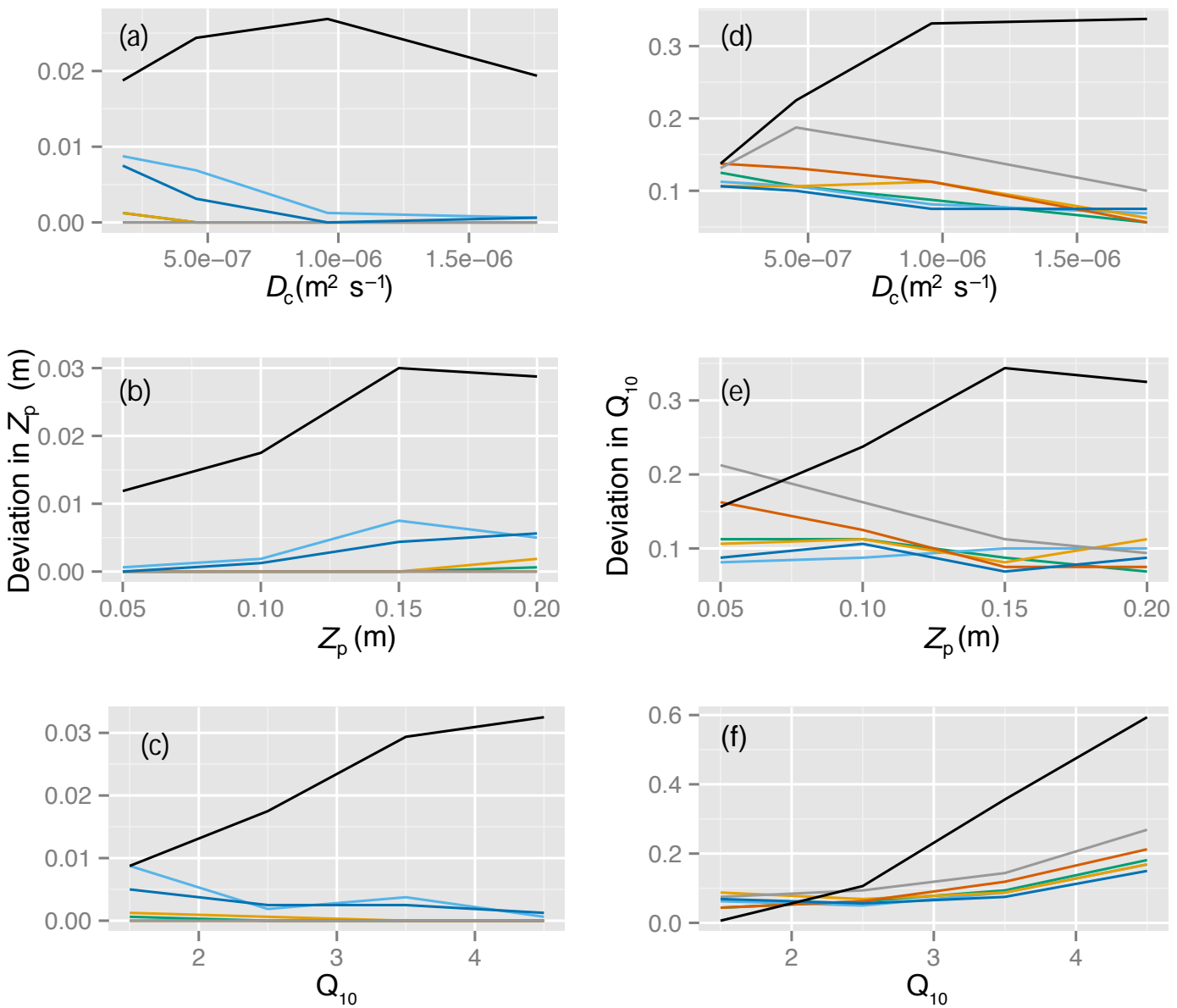

Figure 4. Error in $\mathrm{Q}_{10}$ and $Z_{\mathrm{p}}$ as a function of $\mathrm{Q}_{10}(\mathbf{c}, \mathbf{f}), Z_{\mathrm{p}}(\mathbf{b}, \mathbf{e})$ and $D_{\mathrm{c}}(\mathbf{a}, \mathbf{d})$, for a grouping of the best sensor measurement depth combinations. Individual 5 and $10 \mathrm{~cm}$ observational scenarios are shown in light blue and dark blue, respectively. The $5+15 \mathrm{~cm}$ measurement scenario is shown in green. Orange and red illustrate sensitivity of the $5+10+15$ and $5+10+30 \mathrm{~cm}$ scenarios, respectively. Finally, the 4-point $5+15+30+60 \mathrm{~cm}$ measurement sensitivity is represented in grey while the surface-flux scenario is shown in black. For these sensitivity tests, the known $\mathrm{Q}_{10}$ was 2.0 , and a $Z_{\mathrm{p}}$ of $0.2 \mathrm{~m}$ was used.

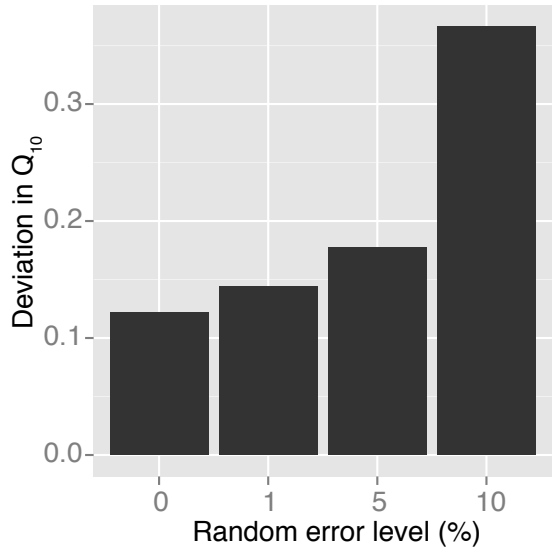

Figure 5. Sample of random error effects on inversion, constrained by one concentration measurement at $5 \mathrm{~cm}$. For this sensitivity test, the known $\mathrm{Q}_{10}$ was 2.0.
As sensors in the field are typically uncertain by 1 to $5 \%$, the inversion method remains feasible. We can thus conclude that the inversion process is rather tolerant of error in measurement.

\subsection{Multi-parameter error landscape}

It is worth investigating in detail the error landscape of the inversion process using a multi-parameter sensitivity tests. For this test, we chose the combination of measurements at 5,10 , and $15 \mathrm{~cm}$, which had resulted in the most accurate inversions on average.

The results from the sensitivity tests are shown in Fig. 6, panels a to $f$. In all combinations, the error in $Z_{\mathrm{p}}$ was very small, with the maximum error for any single inversion being just over $2 \%$. Despite this small error, it remains evident which soil conditions should be avoided for most accuracy. Sites with low diffusivity, production deep in the soil and low $\mathrm{Q}_{10}$ are the most problematic. This is consistent with the 


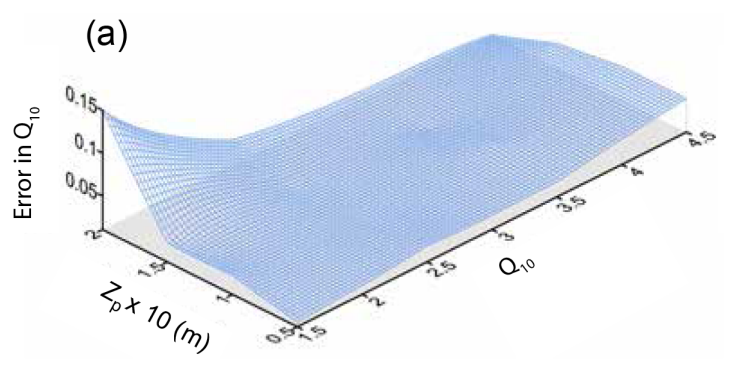

(c)
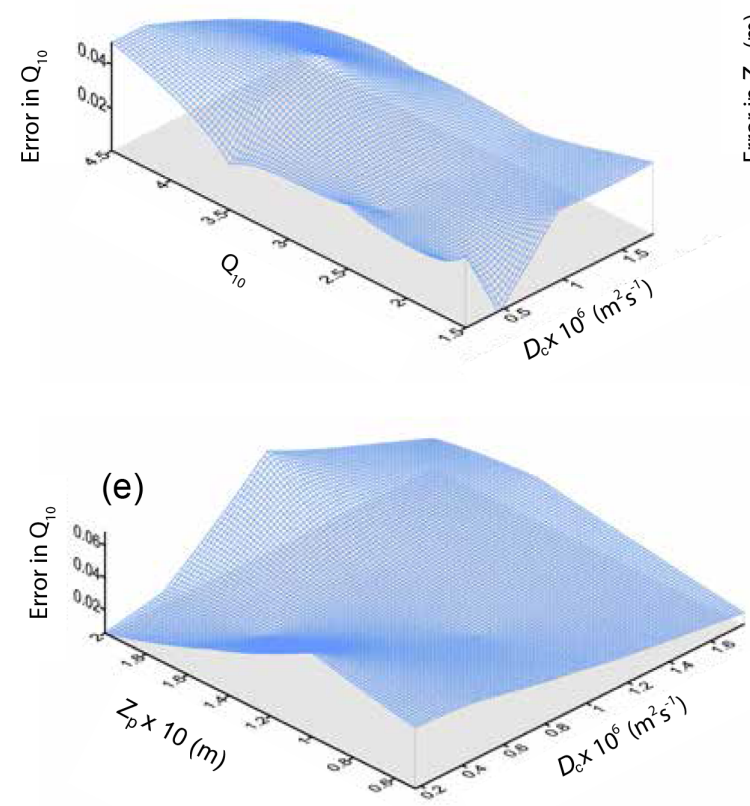

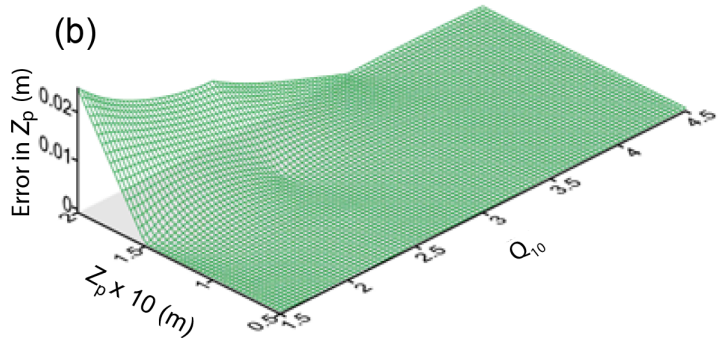

(d)
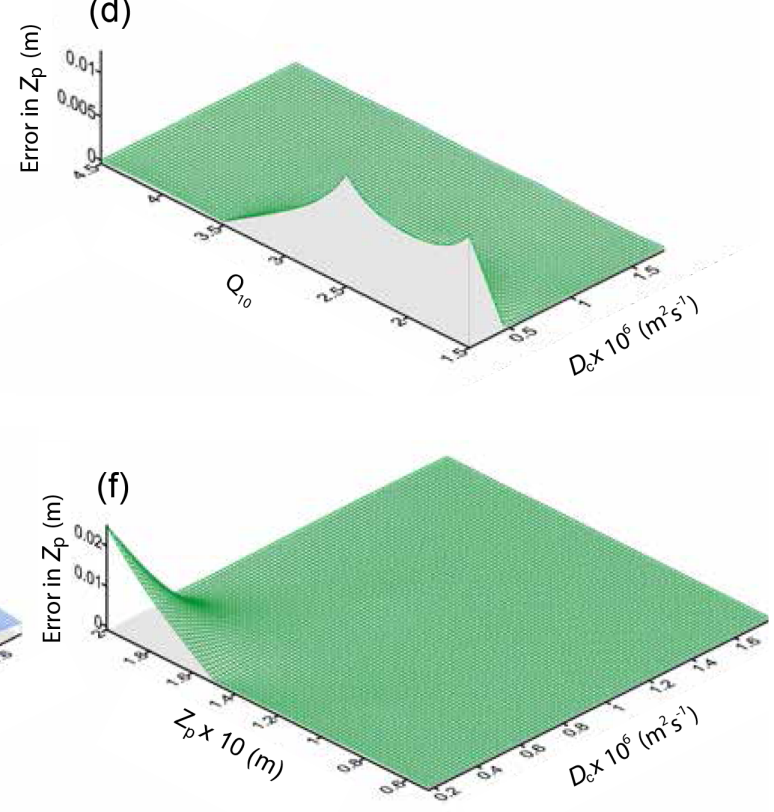

Figure 6. Error in $\mathrm{Q}_{10}$ and $Z_{\mathrm{p}}$ as a function of $\mathrm{Q}_{10}, Z_{\mathrm{p}}$, and diffusivity for the constraint $5+10+15 \mathrm{~cm}$. For these sensitivity tests, the known $\mathrm{Q}_{10}$ was 2.0, and a $Z_{\mathrm{p}}$ of $0.2 \mathrm{~m}$ was used.

results from Fig. 4a, b, and c. Trends were not as evident for error in $\mathrm{Q}_{10}$. In panels a, c, and e the most notable error was found in panel a for high $Z_{\mathrm{p}}$, low $\mathrm{Q}_{10}$. There is an error in $\mathrm{Q}_{10}$ here of almost $15 \%$, which equates to a deviation in $\mathrm{Q}_{10}$ of about 0.225 from its actual value. This result is not unreasonable, but it is significantly higher than results from the other inversions. Plot e demonstrates an interesting result, where there seems to be a valley in the $\mathrm{Q}_{10}$ error, illustrating a tradeoff between $Z_{\mathrm{p}}$ and diffusivity. This is not evident in the other plots, and does not have an intuitive physical explanation. The effect of $\mathrm{Q}_{10}$ on inversion varies, but success hinges quite clearly on soil diffusivity and depth of $\mathrm{CO}_{2}$ production. Choosing a site in the appropriate ranges of these two parameters will maximize chances of success.

\subsection{Limitations and opportunities}

There are both limitations and future opportunities for the inversion approach. In general, the better an inversion is constrained with data, the better it will do in returning the true value for parameters of interest. Some of the soil parameters are distributed in ways that must be assumed. For example, the distribution of $\mathrm{CO}_{2}$ production may be unknown. While most studies in the history of soil modelling have assumed an exponential distribution (and it has been seen in many studies using the gradient technique), some other considerations might help determine whether additional parameterization measurements are needed. For example, knowledge of rooting depth could be one aid. On average, root respiration accounts for $50 \%$ of soil respiration (Hanson et al., 2000) and Jackson et al. (1996) provides root distributions for different terrestrial biomes. Jackson et al. (1996) found that tundra, boreal forest, and temperate grasslands had upwards of 80 $90 \%$ of roots within the top $30 \mathrm{~cm}$ of soil, whereas deserts and temperate coniferous forests had much deeper rooting profiles, with only $50 \%$ of roots within the top $30 \mathrm{~cm}$. These and other methods may be helpful in providing constraint data when running inversions on real time series.

A future opportunity for inversion studies is to determine depth-specific $\mathrm{Q}_{10}$ values, which would be of interest to many researchers. Currently there are few field examples where researchers have determined in situ $\mathrm{Q}_{10}$ as a func- 
tion of depth, but two examples of such gradient studies include Risk et al. (2008) and Tang et al. (2003). Incubations might seem useful in this regard, but are disputed as a representation of in situ conditions, especially at depth (Risk et al., 2008). Ideally, an inversion approach could determine depth-specific $\mathrm{Q}_{10}$, but the reality is challenging. If an additional $100 \mathrm{Q}_{10}$ values were included as unknowns (one for each layer), it would increase computational demand by 100 times. The inversion results would also be confusing, and extreme values at one depth could potentially cause a spurious match to the measured data. The number of non-unique and implausible solutions would rise significantly as a result. A more reasonable approach might be to define $\mathrm{Q}_{10}$ as we do $Z_{\mathrm{p}}$, which is as a function of soil depth. This would be computationally compact, but it is not well known whether a function would be realistic because there are so few examples of $\mathrm{Q}_{10}$-depth profiles against which we could evaluate this approach. The best approach would be to use many profile concentration sensors for constraining data, so that the $e$-folding depth of production could be known, and the inversion could focus instead solely on determining layer-specific $Q_{10}$ values.

Despite the relatively nascent stage of our soil $\mathrm{CO}_{2}$ inversion approach, indications are that it has better theoretical validity than traditional regression approaches, which do not take thermal- and gas-transport lags into account. Our error results here compare very favourably against error analyses generated from detailed examination of regression approaches across thermal- and gas-transport parameter space (Zhang et al., 2015; Phillips et al., 2011; Graf et al., 2008).

\section{Conclusion}

Overall, this inversion method proved successful in testing on synthetic data. Depending on the tolerable level of error for a given application, almost every tested combination resulted in reasonably accurate returned $\mathrm{Q}_{10}$ and $Z_{\mathrm{p}}$ values. The subsurface concentration measurements that yielded the highest error were typically those that would be of least convenience to install and maintain deep in the soil profile. The other constraint associated with high overall error was $\mathrm{CO}_{2}$ surface flux, which would likely be the data with the highest availability. Most of the error from this constraint arises in estimating the $Z_{\mathrm{p}}$ parameter. The $\mathrm{CO}_{2}$ surface flux is still a reasonable means of estimating $\mathrm{Q}_{10}$ values via inversion. While in most cases the error was lower for high diffusivity, shallow production soils, the application of this method is not limited to such regions.

This method is computationally intensive as it performs a sweep through all possible combinations in parameter space. This study used roughly 2.5 core-years of time despite the fact that synthetic time series were short. This full sweep ensures that the global minimum in the objective function is located every time, and when solving inversely for two un- known parameters (as we are), this is not an unreasonable approach. However, if it was of interest in the future to examine longer time series, or additional parameters such as the depth dependence of $\mathrm{Q}_{10}$, resulting in additional unknown parameters, it may be beneficial to explore other search algorithms to increase efficiency, such as simulated annealing.

The next step for this work would be to perform inversions on real time series with appropriate measurement constraints, to obtain temperature sensitivity and $\mathrm{CO}_{2}$ production distribution estimates for various sites. With the increasing availability of high-frequency soil data, there would be no shortage in data to analyse. Applying this method for periods of varying constant moisture levels could also help build an understanding of moisture effects on temperature sensitivity of respiration.

Acknowledgements. The authors wish to thank the Natural Sciences and Engineering Research Council (NSERC) and the Atlantic Computational Excellence Network (ACENet) for project resources. We also wish to thank Nick Nickerson and Chance Creelman for valuable comments early on in this research.

Edited by: A. Ibrom

\section{References}

Cerling, T. E.: The stable isotopic composition of modern soil carbonate and its relationship to climate, Earth Planet. Sc. Lett., 71, 229-240, 1984.

Davidson, E. A., Belk, E., and Boone, R. D.: Soil water content and temperature as independent or confound factors controlling soil respiration in a temperate mixed hardwood forest, Glob. Change Biol., 4, 217-227, 1998.

Davidson, E. A., Janssens, I. A., and Luo, Y.: On the variability of respiration in terrestrial ecosystems: Moving beyond $\mathrm{Q}_{10}$, Glob. Change Biol., 12, 154-164, doi:10.1111/j.13652486.2005.01065.x, 2006.

Graf, A., Weihermüller, L., Huisman, J. A., Herbst, M., Bauer, J., and Vereecken, H.: Measurement depth effects on the apparent temperature sensitivity of soil respiration in field studies, Biogeosciences, 5, 1175-1188, doi:10.5194/bg-5-1175-2008, 2008.

Hanson, P. J., Edwards, N. T., Garten, C. T., and Andrews, J. A.: Separating root and soil microbial contributions to soil respiration: A review of methods and observations, Biogeochemistry, 48, 115-146, 2000.

Jackson, R. B., Canadell, J., Ehleringer, J. R., Mooney, H. A., Sala, O. E., and Schulze, E. D.: A global analysis of root distributions for terrestrial biomes, Oecologia, 108, 389-411, 1996.

Lavoie, M., Phillips, C. L., and Risk, D.: A practical approach for uncertainty quantification of high-frequency soil respiration using forced diffusion chambers, J. Geophys. Res.-Biogeo., 120, 128-146, 2015.

Lloyd, J. and Taylor, J. A.: On the temperature dependence of soil respiration, Funct. Ecol., 8, 315-323, 1994. 
Luo, Y., Wan, S., Hui, D., and Wallace, L. L.: Acclimatization of soil respiration to warming in a tall grass prairie, Nature, 413, 622-625, 2001.

Mahecha, M. D., Reichstein, M., Carvalhais, N., Lasslop, G., Lange, H., Seneviratne, S. I., Vargas, R., Ammann, C., Arain, M. A., Cescatti, A., Janssens, I. A., Migliavacca, M., Montagnani, L., and Richardson, A. D.: Global Convergence in the Temperature Sensitivity of Respiration at Ecosystem Level, Science, 329, 838-840, 2010.

Meir, P., Cox, P., and Grace, J.: The influence of terrestrial ecosystems on climate, Trends Ecol. Evol., 21, 254-260, doi:10.1016/j.tree.2006.03.005, 2006.

Millington, R. J.: Gas diffusion in porous media, Science, 130, 100$102,1959$.

Nickerson, N. and Risk, D.: Physical Controls on the Isotopic Composition of Soil Respired and $\mathrm{CO}_{2}$, J. Geophys. Res.-Biogeo., 114, G01013, doi:10.1029/2008JG000766, 2009.

Phillips, C. L., Nickerson, N., Risk, D., and Bond, B. J.: Interpreting diel hysteresis between soil respiration and temperature, Glob. Change Biol., 17, 515-527, doi:10.1111/j.13652486.2010.02250.x, 2011.

R Core Team: R: A language and environment for statistical computing, R Foundation for Statistical Computing, Vienna, Austria, ISBN 3-900051-07-0, http://www.R-project.org, last access: March 2015.

Raich, J. W., Potter, C. S., and Bhagawati, D.: Interannual variability in global soil respiration, Glob. Change Biol., 8, 800-812, 2002 .
Reichstein, M., Tenhunen, J. D., Roupsard, O., Ourcival, J.-M., Rambal, S., Dore, S., and Valentini, R.: Ecosystem respiration in two Mediterranean evergreen Holm oak forests: Drought effects and decomposition dynamics, Funct. Ecol., 16, 27-39, 2002.

Risk, D., Kellman, L., Beltrami, H., and Diochon, A.: In-situ incubations by root exclusion highlight the climatic sensitivity of soil organic matter pools, Environ. Res. Lett., 3, 044004, doi:10.1088/1748-9326/3/4/044004, 2008.

Tang, J., Baldocchi, D. D., Qi, Y., and Xu, L.: Assessing soil $\mathrm{CO}_{2}$ efflux using continuous measurements of $\mathrm{CO}_{2}$ profiles in soils with small solid-state sensors, Agr. Forest Meteorol., 118, 207220, 2003.

Taylor, B. R., Parkinson, D., and Parsons, W. F. J.: Nitrogen and lignin content as predictors of litter decay rates: A microcosm test, Ecology, 70, 97-104, 1989.

Wan, S. and Luo, Y.: Substrate regulation of soil respiration in a tallgrass prairie: Results of a clipping and shading experiment, Global Biogeochem. Cy., 17, 1054, doi:10.1029/2002GB001971, 2003.

Zhang, Q., Katul, G. G., Oren, R., Daly, E., Manzoni, S., and Yang, D.: The hysteresis response of soil $\mathrm{CO}_{2}$ concentration and soil respiration to soil temperature, J. Geophys. Res.-Biogeo., 120, 1605-1618, 2015.

Zhou, T., Shi, P., Hui, D., and Luo, Y.: Global pattern of temperature sensitivity of soil heterotrophic respiration $\left(\mathrm{Q}_{10}\right)$ and its implications for carbon-climate feedback, J. Geophys. Res.-Biogeo., 114, G02016, doi:10.1029/2008JG000850, 2009. 